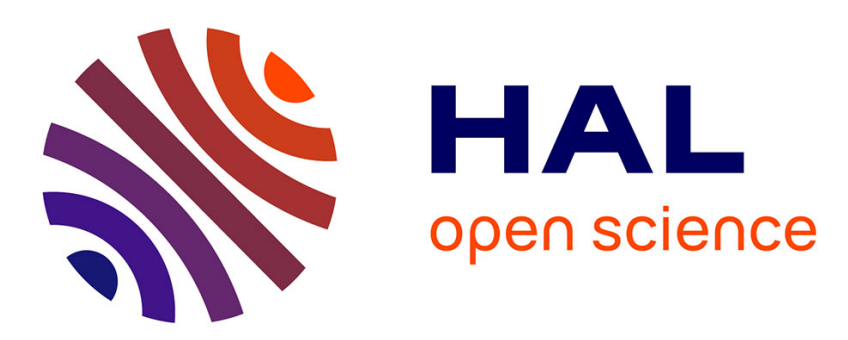

\title{
Lipid remodelling of glycosylphosphatidylinositol (GPI) glycoconjugates in procyclic form trypanosomes: biosynthesis and processing of GPIs revisited
}

Peter Bütikofer, Eva Greganova, Yuk-Chien Liu, Ian J Edwards, Mike J

Lehane, Alvaro Acosta-Serrano

\section{To cite this version:}

Peter Bütikofer, Eva Greganova, Yuk-Chien Liu, Ian J Edwards, Mike J Lehane, et al.. Lipid remodelling of glycosylphosphatidylinositol (GPI) glycoconjugates in procyclic form trypanosomes: biosynthesis and processing of GPIs revisited. Biochemical Journal, 2010, 428 (3), pp.409-418. 10.1042/BJ20100229 . hal-00486863

\section{HAL Id: hal-00486863 https://hal.science/hal-00486863}

Submitted on 27 May 2010

HAL is a multi-disciplinary open access archive for the deposit and dissemination of scientific research documents, whether they are published or not. The documents may come from teaching and research institutions in France or abroad, or from public or private research centers.
L'archive ouverte pluridisciplinaire HAL, est destinée au dépôt et à la diffusion de documents scientifiques de niveau recherche, publiés ou non, émanant des établissements d'enseignement et de recherche français ou étrangers, des laboratoires publics ou privés. 


\title{
Lipid remodelling of glycosylphosphatidylinositol (GPI) glycoconjugates in
} procyclic form trypanosomes: biosynthesis and processing of GPIs revisited

\author{
Peter Bütikofer*1, Eva Greganova*, Yuk-Chien Liu ${ }^{\dagger}$, Ian J. Edwards', Mike J. Lehane ${ }^{\natural}$ \\ and Alvaro Acosta-Serrano ${ }^{\dagger 2}$
}

From the * Institute of Biochemistry and Molecular Medicine, University of Bern, Bühlstrasse 28, 3012 Bern, Switzerland, 'Wellcome Trust Centre for Molecular Parasitology, University of Glasgow, Glasgow G12 8TA, United Kingdom ${ }^{\star}$ Shimadzu Biotech, Wharfside, Trafford Wharf Road, Manchester, M17 1GP, United Kingdom, and the "ILiverpool School of Tropical Medicine, Pembroke Place, Liverpool L3 5QA, United Kingdom.

Short title: GPI lipid remodelling in procyclic form trypanosomes

${ }^{1}$ To whom correspondence may be addressed. Tel.: +4131631 4113; Fax: +41 31631 3737; E-mail: peter.buetikofer@mci.unibe.ch

\footnotetext{
${ }^{2}$ To whom correspondence may be addressed. Present address: Liverpool School of Tropical Medicine, Pembroke Place, Liverpool L3 5QA, United Kingdom. Tel.: +44 151705 2528; Fax: +44 151705 3370; E-mail: a.f.acosta-serrano@liverpool.ac.uk
} 


\section{SYNOPSIS}

The African trypanosome, Trypanosoma brucei, has been used as a model to study the biosynthesis of glycosylphosphatidylinositol (GPI) anchors. In mammalian (bloodstream) form parasites, diacyl-type GPI precursors are remodelled in their lipid moieties before attachment to variant surface glycoproteins. In contrast, the GPI precursors of insect (procyclic) form parasites, consisting of inositol-acylated acyl-lyso-phosphatidylinositol (lyso-(acyl)PI) species, remain unaltered before protein attachment. By using a combination of metabolic labelling, cell-free assays and complementary mass spectrometry analyses, we now show that GPI-anchored glycoconjugates in T. congolense procyclic forms initially receive tri-acylated GPI precursors, which are subsequently de-acylated either at the glycerol backbone or on the inositol ring. Chemical and enzymatic treatments of $\left[{ }^{3} \mathrm{H}\right]$ myristatelabelled lipids in combination with ESI-MS/MS and MALDI-QIT-TOF-MS ${ }^{3}$ analyses indicate that the structure of the lipid moieties of steady-state GPI lipids from $T$. congolense procyclic forms consist of a mixture of lyso-(acyl)PI, diacyl-PI and diacyl-(acyl)PI species. Interestingly, some of these species are myristoylated at the $s n-2$ position. To our knowledge, this is the first demonstration of lipid remodelling at the level of protein- or polysaccharidelinked GPI anchors in procyclic form trypanosomes.

Keywords: Glycosylphosphatidylinositol, Trypanosome, Lipid Biosynthesis, Lipid Remodelling, Posttranslational Modification, Mass Spectrometry.

Abbreviations used: GPI, glycosylphosphatidylinositol; ESI-MS, electrospray ionizationmass spectrometry; ESI-MS-CID-MS, collision induced dissociation and tandem mass spectrometry; CESP, congolense epimastigote-specific protein; PRS, protease resistant surface molecule; PI, phosphatidylinositol; GARP, glutamic acid repetitive protein; JBAM, Jack bean $\alpha$-mannosidase; PI-PLC, phosphatidylinositol-specific phospholipase C; GPI-PLD, GPI-specific phospholipase D; MALDI-QIT-TOF-MS, matrix-assisted laserdesorption/ionization-quadrupole ion trap-time-of-flight mass spectrometry; PA, phosphatidic acid; TLC, thin layer chromatography. 
Bütikofer et al.

\section{INTRODUCTION}

During their life cycles, which alternate between the mammalian bloodstream and the tsetse fly vector, African trypanosomes change the composition of their glycosylphosphatidylinositol (GPI)-anchored surface coats to adapt to new environments [13]. Interestingly, this change in surface coat during differentiation is accompanied by a switch in the types of GPI anchors they express. In T. brucei bloodstream forms, the GPI glycan core structure of variant surface glycoproteins (VSG), consisting of ethanolamine-phosphate-6mannose- $\alpha 1,2$-mannose- $\alpha 1,6$-mannose- $\alpha 1,4$-glucosamine- $\alpha 1,6$-myo-inositol-1-phospholipid, is substituted with a short galactose branch $[4,5]$. In contrast, in the procyclic stage, the GPI core of the procyclins is decorated with the largest and most complex GPI carbohydrate structure known today, consisting of a series of sialylated, branched $\mathrm{N}$-acetyllactosamine $/ \mathrm{N}$ lactobiose repeats [6-8]. Moreover, while the lipid moiety of T. brucei bloodstream form GPI anchors consists exclusively of dimyristoyl phosphatidylinositol (PI) [4, 9], that of procyclic trypanosomes is composed of inositol-acylated acyl-lyso-PI (lyso-(acyl)PI) species with mostly stearic acid $\left(\mathrm{C}_{18: 0}\right)$ in the $s n-1$ position and a heterogeneous fatty acyl chain bound to the $\mathrm{C} 2$ of the inositol ring $[7,10,11]$.

Structural differences in GPI lipid composition between bloodstream and procyclic stages of $T$. brucei are the result of different lipid remodelling reactions that take place before the addition of the GPIs to their respective proteins [12]. In both life cycle stages, GPI synthesis proceeds by stepwise addition of individual core components to PI. Additionally, in both bloodstream and procyclic form trypanosomes, acylation of the inositol ring occurs after the transfer of the first Man residue to GlcN-PI and the resulting inositol-acylated and nonacylated GPI precursors remain in dynamic equilibrium until the addition of the terminal phosphoethanolamine. However, before being transferred to protein, the GPI precursor in bloodstream forms is remodelled and inositol-deacylated to form glycolipid A [13], or P2 [14], whereas in procyclic forms it is deacylated at the $s n-2$ position of the glycerol to yield PP1, a GPI precursor containing a lyso-(acyl)PI lipid $[6,10,11]$. In T. brucei bloodstream forms, acyl chain remodelling of the GPI precursor involves deacylation and reacylation reactions in the endoplasmic reticulum, leading to replacement of the two fatty acyl chains by myristic acid [15, 16]. A further remodelling reaction occurring on protein-bound GPIs, known as myristate exchange, ensures that myristic acid remains the only fatty acid attached to the VSG anchor [17]. In contrast, no lipid remodelling reactions on GPI precursors or 
protein-bound GPI anchors have been described in T. brucei procyclic forms [12].

GPI lipid remodelling has also been reported in other organisms, such as yeast [18], Trypanosoma cruzi [19, 20] and mammalian cells [21, 22], and some of the enzymes involved in the exchange reactions have recently been identified [23-26]. Interestingly, the observation that GPI anchors are often composed of two identical fatty acyl or alcohol chains and, thus, distinctly differ from the lipid composition of bulk PI [21, 27-31], implies that GPI lipid remodelling reactions are a widespread phenomenon.

Trypanosoma congolense is the main causative agent of Nagana, a devastating disease that affects cattle raised in most parts of sub-Saharan Africa. In the tsetse fly host, this parasite is covered by a dense layer of GPI-anchored molecules (Fig. 1). These include glutamic acid- and alanine-rich protein (GARP) [32, 33], EPGENGT procyclins [34], protease-resistant surface molecule (PRS; presumably of polysaccharidic nature) [35], and congolense epimastigote-specific protein (CESP) [36]. Unlike in T. brucei procyclic forms, very little is known about the composition and biosynthesis of GPI-anchored glycoconjugates in procyclic form $T$. congolense. Previous mass spectrometric analyses have shown that the GPI anchors in $T$. congolense procyclic forms consist mainly of lyso-(acyl)PI [34, 35, 37] (see Fig 1). However, it has also been noted that up to $20 \%$ of radioactivity released from $\left[{ }^{3} \mathrm{H}\right]$ myristate-labelled GPI molecules after treatment with GPI-specific phospholipase D (GPI-PLD) was not lyso-phosphatidic acid (lyso-PA), suggesting that a different lipid moiety may be attached to a subpopulation of GPI molecules [35]. In addition, significant amounts of the same labelled material were released by PI-hydrolyzing phospholipase C (PI-PLC), indicating that some of the GPI anchors were not inositol-acylated [35]. Moreover, recent analysis of the lipid portion of purified PRS indicated that it is composed of tri-acylated PIs [38]. Together, these findings suggest that some GPI anchors in T. congolense consist of lipids other than the previously described lyso-(acyl)PI species.

Here we show that $T$. congolense procyclic forms express GPI-anchored surface molecules with different type of PI moieties. The reason for such heterogeneity is that, unlike T. brucei procyclic forms, T. congolense procyclic trypanosomes use tri-acylated GPI precursors, which then undergo a series of de-acylation and re-acylation reactions after they have been transferred to protein. To our knowledge, this is the first demonstration of lipid remodelling reactions occurring on protein- or polysaccharide-bound GPIs in procyclic trypanosomes. 


\section{EXPERIMENTAL}

Unless otherwise specified, all reagents were of analytical grade and from Merck (Darmstadt, Germany), Sigma-Aldrich (Buchs, Switzerland) or MP Biomedicals (Tägerig, Switzerland). $\left[9,10(\mathrm{n})-{ }^{3} \mathrm{H}\right]$ myristic acid $\left(\left[{ }^{3} \mathrm{H}\right]\right.$ myristate, $\left.60 \mathrm{Ci} \mathrm{mmol}^{-1}\right)$, GDP- $\left[{ }^{3} \mathrm{H}\right]$ mannose (GDP- $\left[{ }^{3} \mathrm{H}\right] \mathrm{Man}$, 20-30 Ci mmol ${ }^{-1}$ ) were purchased from American Radiolabelled Chemicals Inc. (St. Louis, MO). Kodak MBX films were from Kodak (Lausanne, Switzerland) and BioMax MS films from GE Healthcare (Buckinghamshire, UK).

\section{Trypanosomes and culture condition}

Procyclic forms of $T$. congolense Kilifi STIB745 and T. congolense Savannah TREU1457 were provided by the Swiss Tropical Institute (Basel, Switzerland) and cultured at $27{ }^{\circ} \mathrm{C}$ in a 1:1 mixture of SM and SDM-79, containing 15\% heat-inactivated foetal bovine serum (Gibco BRL, Basel, Switzerland) [34]. Procyclic forms of T. brucei 427, Antat 1.1 and EP/GPEET knock out mutants [39] were cultured at $27{ }^{\circ} \mathrm{C}$ in SDM-79 [40] containing $15 \%$ heatinactivated foetal bovine serum.

\section{Metabolic labelling and isolation of GPI-anchored molecules}

Metabolic labelling of trypanosomes with $\left[{ }^{3} \mathrm{H}\right]$ myristate was performed as previously described [41]. Briefly, labelled precursors were added to procyclic form trypanosomes at a density of 0.7-1.0 × $10^{7}$ cells $/ \mathrm{ml}$ and incubations were continued for various times (2-48 hours). Cells were spun down, washed with ice-cold buffer (10 mM Tris, $144 \mathrm{mM} \mathrm{NaCl}, \mathrm{pH}$ 7.4), and sequentially extracted with $2 \times 10 \mathrm{ml}$ chloroform:methanol (CM; 2:1, v/v) to extract bulk phospholipids, followed by 3 x $5 \mathrm{ml}$ chloroform:methanol:water (CMW; 10:10:3, v/v/v) to solubilize GPI precursors and free GPIs. The resulting pellet was further extracted with $2 \mathrm{x}$ $0.5 \mathrm{ml}$ of $9 \%(\mathrm{v} / \mathrm{v})$ butan-1-ol to extract GPI-anchored proteins [6, 41]. CMW fractions were pooled, dried under nitrogen and partitioned between butan-1-ol (CMWbut) and water (CMWaqu) to separate GPI lipid precursors and free GPIs, respectively [39]. Incorporation of $\left[{ }^{3} \mathrm{H}\right]$ myristate into the GPI-glycoconjugates extracted with $9 \%$ butan-1-ol, proved to be 10 times more efficient than $\left[{ }^{3} \mathrm{H}\right]$ palmitate (unpublished).

\section{Enzymatic and chemical treatment of protein- and polysaccharide-bound GPI anchors}

$\left[{ }^{3} \mathrm{H}\right]$ myristate-labelled extracts were subjected to enzymatic treatment with PI-PLC from Bacillus thuringiensis (Invitrogen) or purified GPI-PLD from bovine serum as described before [42]. Briefly, dried extracts were incubated in Triton X-100-containing buffer with PI- 
PLC or GPI-PLD for 2 hours at $37{ }^{\circ} \mathrm{C}$. The reaction was stopped by extracting the products twice with water-saturated butan-1-ol. Nitrous acid and mild base treatment was performed as described elsewhere [43]. The cleavage efficiency of each reaction was determined by counting aliquots of the aqueous and butan-1-ol phases. The butan-1-ol phases were pooled and stored at $-20^{\circ} \mathrm{C}$.

\section{Thin layer chromatography (TLC)}

To analyze the $\left[{ }^{3} \mathrm{H}\right]$-labelled reaction products after PI-PLC, GPI-PLD or nitrous acid treatment, extracts were applied to silica gel 60 plates (Merck) and separated by TLC using solvent system 1, composed of chloroform:methanol:acetic acid:water (25:15:4:2, v/v/v/v). $\left[{ }^{3} \mathrm{H}\right]$-labelled monoacylglycerol and diacylglycerol were separated using solvent system 2 , composed of benzene:diethyl ether:ethanol:acetic acid (50:40:2:0.2, v/v/v/v). Appropriate lipid standards were run alongside the samples on each plate. Radioactivity was detected by scanning the air-dried plates with a radioisotope detector (Berthold Technologies, Regensdorf, Switzerland) and quantified using the Rita Star® software provided by the manufacturer. Alternatively, the plates were sprayed with $\mathrm{En}^{3}$ hance (Perkin Elmer) and exposed to MXB film at $-70{ }^{\circ} \mathrm{C}$. Lipid spots on TLC plates were visualized by exposure to iodine vapour.

\section{Mass spectrometry analysis}

Butan-1-ol extracts containing GARP, EPGENGT procyclin and PRS (equivalent to $\sim 5 \times 10^{8}$ parasites) from parasites labelled with $\left[{ }^{3} \mathrm{H}\right]$ myristate for 6 or 48 hours were washed five times with butan-1-ol and water (to eliminate phospholipid contaminants), dried in a speed-vac and submitted to nitrous acid deamination as described [43]. The released PI species were recovered as above, small quantities were then transferred to Water nanoflow tips (Millipore) and analyzed in negative ion electrospray ionization-mass spectrometry (ESI-MS) on a ABI Q-StarXL with tip and declustering potentials of 900 and $60 \mathrm{~V}$, respectively. Collision induced dissociation and tandem mass spectra (ESI-MS-CID-MS) were collected in product ion scanning mode using collision voltages of 25 to $60 \mathrm{~V}$. 
Bütikofer et al.

\section{RESULTS}

In vivo $\left[{ }^{3} \mathrm{H}\right]$ myristate-labelling reveals the presence of GPI-anchored molecules with triacylated lipid moieties in $T$. congolense procyclic forms

Previous reports suggested that at least part of the GPI anchors in T. congolense procyclic forms may consist of alternatively acylated PI species. To corroborate these observations, we first pulse-labelled live T. brucei (as control parasites) and T. congolense procyclic forms with $\left[{ }^{3} \mathrm{H}\right]$ myristate. The GPI anchors from $T$. brucei procyclic forms have been shown to consist exclusively of lyso-(acyl)PI species [7, 11, 34, 37]. In agreement with these reports, we found that in a butan-1-ol extract from $T$. brucei procyclic forms labelled with $\left[{ }^{3} \mathrm{H}\right]$ myristate for 40 hours, treatment by GPI-PLD released 55-64\% (range of 6 independent experiments) of radioactivity from GPI-anchored proteins (mostly procyclins), all of which migrated as lyso-PA (Fig. 2A, left). Furthermore, only trace amounts of label (1-3\%; range of 3 experiments) were released by PI-PLC (Fig. 2A, right). In contrast, treatment of $\left[{ }^{3} \mathrm{H}\right]$ myristate-labelled GPI molecules from T. congolense Savannah and Kilifi procyclic forms with GPI-PLD released 81-96\% (range of 8 independent experiments) of radioactivity, which migrated as two distinct spots on TLC (Fig. 2A, left). Consistent with the known lysoPI anchor of T. congolense GARP [37] and EPGENGT procyclin [34], part of the label migrated as lyso-PA. However, a considerable fraction of radioactivity co-migrated with a PA standard run on the same TLC plate, indicating that a fraction of GPI-anchored molecules consists of diacyl-type PI. In addition, part of the label (30-49\%; range of 4 experiments) was released from T. congolense GPI molecules by treatment with PI-PLC (Fig. 2A, right).

To study if the relative distribution of label between lyso-PA and PA represents a steady state situation or changes during labelling, T. congolense Savannah and Kilifi procyclic forms were incubated with $\left[{ }^{3} \mathrm{H}\right]$ myristate for 6,12 or 23 hours and the products released by GPI-PLD were analyzed by TLC (Fig. 2B). The results showed that the relative amount of radioactivity in PA is maximal after 6 hours of labelling and decreases with prolonged incubation time, with a concomitant increase in lyso-PA.

To further analyze the composition and structures of the radioactive GPI lipid moieties, T. congolense Savannah procyclic forms were labelled with $\left[{ }^{3} \mathrm{H}\right]$ myristate for 6 or 48 hours and GPI anchors were analysed by enzymatic and chemical treatment followed by TLC separation of the products as above. After 6 hours of labelling, most radioactivity released from GPI molecules by GPI-PLD migrated as PA while the PI-PLC-released material co-migrated with the solvent front (Fig. 3A; see also Fig. 2B). Treatment with 
nitrous acid (which in GPI molecules specifically cleaves between non-acetylated glucosamine and inositol) released two main products, one of which co-migrated with a PI standard (Fig. 3A, left panel), whereas the other migrated closer to the front, consistent with being more hydrophobic and possibly representing tri-acylated PI, i.e. diacyl-(acyl)PI. In contrast, after 48 hours of labelling, a substantial amount of radioactivity released by GPIPLD migrated as lyso-PA (Fig. 3A, right panel). In addition, a third product is seen after nitrous acid treatment. This product migrated between diacyl-PI and diacyl-(acyl)PI and was resistant to cleavage by phospholipase $\mathrm{A}_{2}$ and PI-PLC (data not shown) and, therefore, identified as lyso-(acyl)PI. Finally, the PI-PLC-released products were analyzed in another TLC system to separate monoacylglycerol and diacylglycerol (Fig. 3B). The results showed that all radioactivity from the 6 and 48 hours time points co-migrated with a diacylglycerol standard. In summary, these analyses indicate that GPI-glycoconjugate lipids in $T$. congolense procyclic forms are remodelled over time, from diacyl-(acyl)PI and diacyl-PI species to lyso-(acyl)PI species.

\section{Pulse-chase experiments corroborate lipid remodelling of protein-bound GPIs in $T$. congolense procyclic forms}

To confirm a putative GPI lipid remodelling, T. congolense Savannah procyclic forms were incubated in the presence of $\left[{ }^{3} \mathrm{H}\right]$ myristate for a short time only ( 2 hour pulse) and the labelled GPI anchors chased over a period of 22 hours in the absence of extracellular label. The results showed that after the pulse, almost all radioactivity released from GPI-anchored molecules by GPI-PLD migrated as PA (Fig. 4, left; see also Figs. 2B and 3A). In contrast, after 4 and 22 hours of chase, increasing amounts of label co-migrated with the lyso-PA standard. Interestingly, the $4 \mathrm{~h}$ time point showed two spots in the lyso-PA range, one of which co-migrated with the lyso-PA spot after the 22 hours chase while the other showed a reduced mobility and co-migrated with the minor spot seen after the pulse ( 0 hour chase). This result suggests that the labelled lyso-PA species released at the end of the pulse consist of less hydrophobic, i.e. shorter, acyl chains compared to the lyso-PA species released after the 4 hours chase. A similar experiment with $\left[{ }^{3} \mathrm{H}\right]$ myristate-labelled $T$. brucei procyclic forms showed that all radioactivity co-migrated with lyso-PA during the chase (Fig. 4, right). In summary, the time course and pulse-chase experiments using $\left[{ }^{3} \mathrm{H}\right]$ myristate-labelled extracts indicate that in $T$. congolense procyclic forms, candidate GPI molecules receive triacylated, i.e. inositol-acylated diacyl-type, GPI precursors. Subsequently, both the protein- and polysaccharide-bound anchors are remodelled by de-acylation on the glycerol or inositol 
moiety. The presence of PI-PLC-sensitive GPIs further corroborates that some of these molecules are indeed de-acylated on the inositol.

\section{Mass spectrometry analysis of steady state GPI lipid molecules}

While the above $\left[{ }^{3} \mathrm{H}\right]$ myristate-labelling experiments (Figs. 2-4) provide information on possible lipid remodelling reactions by following trace amounts of radioactivity, they may not reveal the bulk composition of the GPI lipid moiety. Moreover, the changes observed in lipid composition could be accounted for in part by differences in cell cycle or age of the cultures. Therefore, to determine the steady-state GPI lipid composition in $T$. congolense procyclic forms, we submitted butan-1-ol extracts containing GPI molecules from unlabelled cells, which were cultured in parallel to the cells used for $\left[{ }^{3} \mathrm{H}\right]$ myristate labelling experiments (Fig. 3), to nitrous acid deamination and analyzed the released PI species by negative-ion ESI-MS, ESI-CID-MS, MALDI-QIT-TOF-MS and MALDI-QIT-TOF-MS ${ }^{3}$. The results showed that the ESI mass spectra of the released PI species are identical in both butan-1-ol samples and consist mainly of species with $m / z$ 810, 862, 864, 866 and 1072 (Fig. 5 and Fig. $\mathrm{S} 1)$. The collision-induced product spectrum of the major $[\mathrm{M}-\mathrm{H}]^{-}$pseudomolecular ion at $m / z$ of 862 (6 hours sample), suggests that this species corresponds to 1-stearoyl-2-lyso-snglycero-3-phospho-(2'-O-linoleoyl-1'-myo-inositol) (Fig. S2). This preliminary assignment is based on the typical fragmentation pattern indicative for the presence of an acyl group on the inositol ring, i.e. the low intensity of $\mathrm{m} / \mathrm{z}$, ions 223, 241 (for inositol 1,2-cyclic phosphate), $279\left(\mathrm{C}_{18: 2}\right.$ fatty acid $)$, and the key signature ion at $\mathrm{m} / z .503\left(\left(\mathrm{C}_{18: 2}\right)\right.$-inositol 1,2-cyclic phosphate), suggesting that linoleate is attached to inositol. In addition, the high intensities of $m / z$ ions 283 ( $\mathrm{C}_{18: 0}$ carboxylated fragment), 153 (glycerol-2,3-cyclic phosphate) and 419 (1stearoyl-sn-glycerol-2,3-cyclic phosphate) indicate the presence of stearate attached to the $s n$ 1 position of the glycerol backbone. Importantly, however, the $m / z, 577$ ion, which in previous reports has been assigned exclusively as the fragment ion missing stearate at the $s n-1$ position $[7,34,37]$, can also be interpreted as two putative isobaric molecules with the $\mathrm{C}_{18: 2}$ fatty acid linked to either the inositol ring (i.e. glycero-3-phospho-(2'-O-linoleoyl-1'-myo-inositol) or the glycerol backbone (i.e. 1-lyso-2-linoleoyl-sn-glycero-3-phospho-(1'-myo-inositol) or 1linoleoyl-2-lyso-sn-glycero-3-phospho-(1'-myo-inositol), with the former structure being more likely assuming that $\mathrm{C}_{18: 0}$ was originally located at the $s n-1$ position) (see Fig. S2B).

To further investigate the possible presence of two isobaric structures, we analyzed the same samples by tandem mass spectrometry using MALDI-QIT-TOF-MS. CID of the ion at $m / z 862$ produced abundant $[\mathrm{M}-\mathrm{H}]^{-}$product ions similar to that obtained by ESI-MS/MS, 
including the fragment at $m / z 577$ (not shown). When the $m / z 577$ ion was further fragmented, the MALDI-QIT-TOF-MS ${ }^{3}$ product ion spectrum clearly generated $[\mathrm{M}-\mathrm{H}]^{-}$ pseudomolecular ions that corroborate the presence of an inositol-acylated molecule (supplemental Fig. S4A). This is the case of the signature ion at $m / z, 503\left(\left(\mathrm{C}_{18: 2}\right)\right.$-inositol 1,2cyclic phosphate), which further demonstrates that linoleate is attached to inositol. Moreover, and in agreement with previous reports [7, 44], the high intensity of the cyclic glycerophosphate $(\mathrm{m} / \mathrm{z}, 153)$, together with a weak signal for the carboxylated fatty acid ion $(\mathrm{m} / z$ 279), strongly suggests the presence of an inositol-acylated PI species containing a weak carboxylated fatty acid (i.e. $\mathrm{C}_{18: 2}$ ) attached to the 2-position of the inositol ring. On the other hand, the product ion spectrum also supports the presence of a species containing glycerol. In particular, the key ions at $\mathrm{m} / \mathrm{z} 315,297$ and the ion at $\mathrm{m} / \mathrm{z} 153$, corresponding to glycerophosphoinositol, glycero-phosphoinositol - $1 \mathrm{H}_{2} \mathrm{O}$ and glycerol-2,3-cyclic phosphate, respectively, suggest that this isobaric species still retains the glycerol backbone [45].

Together, these results strongly suggest that the ion at $m / z, 862$ likely represents a mixture of two isobaric species: 1-stearoyl-2-linoleoyl-sn-glycero-3-phospho-(1'-myoinositol) and 1-stearoyl-2-lyso-sn-glycero-3-phospho-(2'-O-linoleoyl-1'-myo-inositol). The relative proportions of the two species cannot be determined from these analyses. Almost identical results were obtained in the ESI-MS/MS and MALDI-QIT-TOF-MS ${ }^{3}$ collisional spectra of the $m / z 862$ ion from the 48 hour sample (not shown). In addition, similar product ion spectra where obtained after fragmentations of the ions with $\mathrm{m} / \mathrm{z} 864$ and 866 (not shown). In both cases, the location of the stearoyl chain appears conserved at $s n-1$, but they differ in the type of fatty acids attached to the inositol or the $s n-2$ position of the glycerol $\left(\mathrm{C}_{18: 1}\right.$ oleoyl and $\mathrm{C}_{18: 0}$ stearoyl for $m / z 864$ and 866 ions, respectively). The ESI-MS-CID-MS analysis of the minor ions at $m / z, 810$ (6 hours butan-1-ol extract) and $m / z, 1072$ (Figs. S3 and S5), suggest that both species contain myristate at the $s n-2$ position.

In summary, these results unequivocally demonstrate that the lipid tails of steady-state GPI anchors in T. congolense procyclic forms consist of a mixture of lyso-(acyl)PI, diacyl-PI, and diacyl-(acyl)PI species. An overview on the identified PI molecular species is given in Table 1.

\section{In vitro analysis of the GPI biosynthetic pathway in $T$. congolense procyclic forms}

To corroborate that $T$. congolense procyclic forms make triacylated GPI precursors, we analyzed the GPI biosynthetic pathway of $T$. congolense procyclic forms by pulse labelling washed parasite membranes with GDP- $\left[{ }^{3} \mathrm{H}\right] \mathrm{Man}$ and determined the structure of the GPI 
anchor precursors after chemical and enzymatic cleavage, and TLC analysis of the products. As shown in Figs. S6 and S7, these experiments strongly suggest that procyclic form $T$. congolense membranes synthesize GPI precursors (i.e. PP3- and PP1-like) with the same characteristics as previously described in procyclic form T. brucei (see the supplementary section for a detailed explanation of these experiments).

\section{GPI lipid remodelling in free GPIs from $T$. brucei procyclic forms}

It has been reported that free GPIs from T. brucei procyclic forms consist of a mixture of lyso- and diacyl-type lipids [39]. These results, obtained in mutant parasites lacking the expression of the major GPI-anchored surface proteins EP and GPEET procyclins (EP/GPEET knock out cells), were in contrast to the previously reported exclusive presence of lyso-(acyl)PI in protein-bound GPI anchors in T. brucei procyclic forms [6, 7, 11]. We reinvestigated these findings by incubating EP/GPEET knock out cells in the presence of $\left[{ }^{3} \mathrm{H}\right]$ myristate for 1-48 hours and analyzing the composition of the GPI-PLD-released lipids of free GPIs (Fig. 6). Our results showed that after 1 hour of labelling with $\left[{ }^{3} \mathrm{H}\right]$ myristate, roughly equal amounts of radioactivity were recovered in lyso-PA and PA. In contrast, after labelling trypanosomes for 48 hours, lyso-PA was clearly the dominant product, indicating that the label re-distributed from PA to lyso-PA with increasing labelling time. Thus, in these T. brucei mutant parasites, which express approximately 10-fold more free GPIs than wildtype trypanosomes [39], similar lipid remodelling reactions may be active as in $T$. congolense procyclic forms.

\section{DISCUSSION}

The GPI lipid moieties of surface glycoconjugates from $T$. brucei and $T$. congolense procyclic forms have been known to consist exclusively of lyso-(acyl)PI species [6, 7, 11, 34, $35,37,41]$. These results, which are based on chemical and mass spectrometric analyses, are consistent with the current scheme for GPI biosynthesis in procyclic form T. brucei, where lyso-(acyl)PI precursors are attached to protein ([11, 13, 46], reviewed by [12, 47]). However, prompted by our observations that in T. brucei procyclin null parasites [39] and T. congolense procyclic forms [35] at least a subpopulation of GPI anchors contain different lipid moieties, we re-investigated the lipid structures and biosynthesis of GPIs in procyclic form $T$. congolense. 


\section{A novel type of GPI lipid remodelling in T. congolense procyclic forms}

Our metabolic incorporation and pulse-chase experiments using $\left[{ }^{3} \mathrm{H}\right]$ myristate suggest that most of the GPI acceptor molecules are likely to be first attached to tri-acylated GPI anchors and subsequently become de-acylated. Our cell-free system experiments using GDP- $\left[{ }^{3} \mathrm{H}\right] \mathrm{Man}$ demonstrated that membranes from $T$. congolense procyclics are indeed capable of synthesizing a tri-acylated GPI precursor, whose migration on TLC and structure resembles that of T. brucei PP3. This contrasts with the current model of GPI biosynthesis in T. brucei procyclic forms where a lyso-PI lipid (from the GPI precursor PP1) appears to remain unmodified after its transfer to procyclins. Thus, unlike T. brucei, T. congolense procyclic forms have the capability to remodel their GPI lipids after they have been transferred to protein or polysaccharides (i.e. PRS). Evidence for lipid remodelling of protein-bound GPIs has previously been reported for T. cruzi $[19,20]$ and yeast [48]. In these cases, GPI remodelling involves exchange of the entire lipid moiety, i.e. from PI to inositolphosphoryl ceramide.

Based on our results, we propose the following model for GPI biosynthesis and processing in $T$. congolense procyclic forms (Fig. 7). In a first step, a tri-acylated GPI precursor (PP3-like) is transferred to GPI acceptor molecules. Such a precursor is likely heterogeneous in the type of fatty acids (i.e. $\mathrm{C}_{18: 0}, \mathrm{C}_{18: 1}$ and $\mathrm{C}_{18: 2}$ ) linked to the $s n-2$ position of the glycerol backbone and the inositol ring. Secondly, and possibly during transit between the ER and Golgi, a subpopulation of GPI-anchored molecules, consisting primarily of GARP and EPGENGT procyclin, undergoes de-acylation at the $s n-2$ position of the glycerol backbone, forming GPI anchors with lyso-(acyl)PI species. In addition, our data suggest that a small fraction of these molecules may also undergo de-acylation on the inositol, resulting in the formation of diacyl-type GPI anchors. The presence of these structures was demonstrated by the high amount of PI-PLC-sensitive GPI anchors after short labelling times with $\left[{ }^{3} \mathrm{H}\right]$ myristate, and by MALDI-QIT-TOF tandem mass spectrometry. Clearly, these results are in contrast to the previously published structures of the GARP and EPGENGT procyclin GPI anchors, which showed lyso-(acyl)PI [34, 37]. In these studies, however, the presence of diacyl-PI species may have been missed, probably because of the difficulties in detecting specific fragment ions that would discriminate between lyso-(acyl)PI and diacyl-PI species (many of the $\mathrm{MS}^{2}$ fragment ions are generated by both type of lipids). In this work, these structures could only be demonstrated because of the high sensitivity and precursor ion selection resolution of the Resonance 3D MALDI-QIT-TOF mass spectrometer, which 
produced key fragment ions that made the identification of isobaric PI species in these samples possible. Unfortunately, we were unable to determine the relative proportions between isobaric species as inositol-acyl bonds are much more stable compared to fatty acids in diacyl-PI lipids [7, 44]. However, judging by the intensity of key product ions generated after CID (i.e. especially the $\mathrm{MS}^{3}$ ion at $m / z$ 315, 297 and 153, which support the presence of a glycerolipid), it appears that the diacyl-PI molecules are abundant species in both samples (i.e. 6 and 48 hours). Stronger evidence for the presence of diacyl-GPI molecules in $T$. congolense procyclic forms came from the CID product ion spectra of the ion at $\mathrm{m} / \mathrm{z} 810$ : both the EIS-MS/MS and the MALDI-QIT-TOFMS ${ }^{3}$ analyses of the diagnostic $\left(\mathrm{MS}^{2}\right)$ product ion at $m / z, 581$ unambiguously identified the $[\mathrm{M}-\mathrm{H}]^{-}$pseudomolecular ion $\mathrm{m} / z, 810$ as 1-stearoyl-2-sn-myristoyl-glycero-3-phospho-(1'-myo-inositol).

Interestingly, the diacyl-PI species characterized by mass spectrometry contain myristate, presumably at the $s n-2$ position of the glycerol backbone. This reinforces our recent finding that the GPI anchor of PRS is myristoylated and, in addition, suggests that some of the machinery involved in the addition of myristic acid to the VSG anchor in bloodstream form trypanosomes is likely to be active in the procyclic stage of $T$. congolense as well [38].

Inositol de-acylation of protein bound GPIs in T. congolense may resemble the situation in mammalian cells and yeast, where de-acylation of inositol takes place after transfer of the GPI precursor to protein, a reaction catalyzed by PGAP1 in mammalian cells and Bst1p in yeast [49]. In contrast, it clearly differs from T. brucei, where inositol deacylation occurs during GPI biosynthesis only and is mediated by at least two different enzymes [50, 51].

A question that remains to be investigated is the significance of the production of PP1-like precursor by membranes of $T$. congolense procyclic trypanosomes. Although our in vivo labelling experiments using $\left[{ }^{3} \mathrm{H}\right]$ myristate suggest that PP3-like is the putative GPI precursor in $T$. congolense procyclics, we cannot rule out that minor quantities of PP1-like are also transferred to protein and then quickly acylated on the $s n-2$ position, thus producing tri-acylated PIs (e.g. like those in PRS). However, our lipid analyses from the metabolic and pulse-chase experiments suggest that very little lyso-PIs are present at early time-points of labelling (Figs. 4 and 6), although this process could take place quite rapidly and, therefore, may be difficult to detect. An alternative explanation is that PP1-like GPIs are produced in excess and, thus, may represent catabolic products of PP3-like precursors, similar to the situation occurring between glycolipid A and C in T. brucei bloodstream forms [12, 47]. 
Lastly, another group of molecules appears to maintain the same lipid type originally present in the GPI precursor (i.e. PRS, containing $\mathrm{C}_{18: 1}$ at the sn-2 position [38]). This interpretation is supported by MS analysis of the total pool of cellular PI, showing that the species containing $\mathrm{C}_{18: 1}$ at the $s n-2$ position of the glycerol backbone is most abundant (Bütikofer, P., Greganova, E., M. Serricchio, Liu, Y-C, and Acosta-Serrano, A., manuscript in preparation). Whether the same pool of molecules could also undergo fatty acid remodelling at the $s n-2$ position, thereby forming, for instance, species containing myristate, remains to be demonstrated. In summary, compared to T. brucei, attachment and processing of GPI anchors in $T$. congolense procyclic forms is more complex and results in the formation of multiple types of GPI lipid moieties. It would be interesting to determine the lipid structure of CESP and also to investigate whether the same post-transfer fatty acid remodelling is also operative in the epimastigote form of $T$. congolense.

\section{GPI remodelling in $T$. brucei procyclic forms}

Although wild type T. brucei procyclic forms only use lyso-(acyl)PI to anchor EP $[6,11]$ and GPEET procyclins [7, 41], we confirmed an earlier report [39] showing the presence of diacyl-type lipids in the anchor of free GPIs in mutant parasites lacking the expression of $T$. brucei procyclins. As a result of the deficiency of GPI-anchored coat proteins, EP/GPEET knock out parasites express 10-fold more free GPIs as wild-type trypanosomes [39]. Thus, it is possible that the presence of diacyl-type PIs in free GPIs represents an artefact related to overproduction of free, i.e. unused, GPIs in these cells. Nevertheless, our data clearly demonstrate that $T$. brucei procyclic forms are capable of making diacyl-type GPI structures. Thus, we cannot rule out the possibility that remodelling of protein-bound GPIs also occurs in wild-type T. brucei procyclic forms, but the turnover of intermediates occurs too rapidly to be detected by labelling. Alternatively, T. brucei procyclic forms may use two different GPI precursors, i.e. PP1 for protein attachment and PP3 for free GPIs (Fig. S8). 
Bütikofer et al.

\section{ACKNOWLEDGEMENTS}

We are indebted to Mike Ferguson and Douglas Lamont (University of Dundee) for the generous use of the different mass spectrometers and thank Shimadzu Biotech (Manchester, $\mathrm{UK}$ ) for the use of the Resonance 3D ion trap. We thank Mike Ferguson for helpful discussions, Jennifer Jelk and Monika Rauch for technical assistance during part of the study, and Lee R. Haines and Deirdre Walshe for critical reading of the manuscript. PB thanks Hanery Amman for valuable input.

\section{FUNDING}

This work was supported by Swiss National Science Foundation grant 3100A0-116627 to PB. AA-S was supported by a Research Career Development Fellowship and a VIP Award from the Wellcome Trust. Y-CL was supported by a Wellcome Trust PhD studentship. 


\section{REFERENCES}

1 Roditi, I. and Liniger, M. (2002) Dressed for success: the surface coats of insectborne protozoan parasites. Trends Microbiol. 10, 128-134

2 Pays, E. (2005) Regulation of antigen gene expression in Trypanosoma brucei. Trends Parasitol. 21, 517-520

3 Roditi, I. and Lehane, M. J. (2008) Interactions between trypanosomes and tsetse flies. Curr Opin Microbiol. 11, 345-351

4 Ferguson, M. A. J., Homans, S. W., Dwek, R. A. and Rademacher, T. W. (1988) Glycosylphosphatidylinositol moiety that anchors Trypanosoma brucei variant surface glycoprotein to the membrane. Science. 239, 753-759

5 Mehlert, A., Richardson, J. M. and Ferguson, M. A. J. (1998) Structure of the glycosylphosphatidylinositol membrane anchor glycan of a class-2 variant surface glycoprotein from Trypanosoma brucei. J. Mol. Biol. 277, 379-392

6 Ferguson, M. A. J., Murray, P., Rutherford, H. and McConville, M. J. (1993) A simple purification of procyclic acidic repetitive protein and demonstration of a sialylated glycosyl-phosphatidylinositol membrane anchor. Biochem. J. 291, 51-55

7 Treumann, A., Zitzmann, N., Hülsmeier, A., Prescott, A. R., Almond, A., Sheehan, J. and Ferguson, M. A. J. (1997) Structural characterization of two forms of procyclic acidic repetitive protein expressed by procyclic forms of Trypanosoma brucei. J. Mol. Biol. 269, 529-547

8 Izquierdo, L., Nakanishi, M., Mehlert, A., Machray, G., Barton, G. J. and Ferguson, M. A. (2009) Identification of a glycosylphosphatidylinositol anchor-modifying beta1-3 Nacetylglucosaminyl transferase in Trypanosoma brucei. Mol. Microbiol. 71, 478-491 9 Gerold, P., Striepen, B., Reitter, B., Geyer, H., Geyer, R., Reinwald, E., Risse, H.-J. and Schwarz, R. T. (1996) Glycosyl-phosphatidylinositols of Trypanosoma congolense: Two common precursors but a new protein anchor. J. Mol. Biol. 261, 181-194

10 Field, M. C., Menon, A. K. and Cross, G. A. M. (1991) Developmental variation of glycosylphosphatidylinositol membrane anchors in Trypanosoma brucei. J. Biol. Chem. 266, $8392-8400$

11 Field, M. C., Menon, A. K. and Cross, G. A. M. (1991) A

glycosylphosphatidylinositol protein anchor from procyclic stage Trypanosoma brucei: Lipid structure and biosynthesis. EMBO J. 10, 2731-2739 
12 Ferguson, M. A. J. (1999) The structure, biosynthesis and functions of glycosylphosphatidylinositol anchors, and the contributions of trypanosome research. J. Cell Sci. 112, 2799-2809

13 Masterson, W. J., Doering, T. L., Hart, G. W. and Englund, P. T. (1989) A novel pathway for glycan assembly: Biosynthesis of the glycosyl-phosphatidylinositol anchor of the trypanosome variant surface glycoprotein. Cell. 56, 793-800

14 Menon, A. K., Schwarz, R. T., Mayor, S. and Cross, G. A. M. (1990) Cell-free synthesis of glycosyl-phosphatidylinositol precursors for the glycolipid membrane anchor of Trypanosoma brucei variant surface glycoproteins. J. Biol. Chem. 265, $9033-9042$ 15 Masterson, W. J., Raper, J., Doering, T. L., Hart, G. W. and Englund, P. T. (1990) Fatty acid remodeling: A novel reaction sequence in the biosynthesis of Trypanosome glycosyl phosphatidylinositol membrane anchors. Cell. 62, 73-80 16 Morita, Y. S., Acosta-Serrano, A., Buxbaum, L. U. and Englund, P. T. (2000) Glycosyl phosphatidylinositol myristoylation in African trypanosomes. New intermediates in the pathway for fatty acid remodeling. J. Biol. Chem. 275, 14147-14154

17 Buxbaum, L. U., Milne, K. G., Werbowetz, K. A. and Englund, P. T. (1996) Myristate exchange on the Trypanosoma brucei variant surface glycoprotein. Proc. Natl. Acad. Sci. USA. 93, 1178-1183

18 Sipos, G., Puoti, A. and Conzelmann, A. (1994) Glycosylphosphatidylinositol membrane anchors in Saccharomyces cerevisiae: absence of ceramides from complete precursor glycolipids EMBO J. 13, 2789-2796

19 Acosta-Serrano, A., Schenkman, S., Yoshida, N., Mehlert, A., Richardson, J. M. and Ferguson, M. A. J. (1995) The lipid structure of the glycosylphosphatidylinositol-anchored mucin-like sialic acid acceptors of Trypanosoma cruzi changes during parasite differentiation from epimastigotes to infective metacyclic trypomastigote forms. J. Biol. Chem. 270, 2724427253

20 Bertello, L. E., Alves, M. J., Colli, W. and de Lederkremer, R. M. (2004) Inositolphosphoceramide is not a substrate for the first steps in the biosynthesis of glycoinositolphospholipids in Trypanosoma cruzi. Molecular and biochemical parasitology. 133, 71-80

21 Houjou, T., Hayakawa, J., Watanabe, R., Tashima, Y., Maeda, Y., Kinoshita, T. and Taguchi, R. (2007) Changes in molecular species profiles of glycosylphosphatidylinositol anchor precursors in early stages of biosynthesis. J. Lipid Res. 48, 1599-1606 
22 Kinoshita, T., Fujita, M. and Maeda, Y. (2008) Biosynthesis, remodelling and functions of mammalian GPI-anchored proteins: recent progress. J. Biochem. 144, 287-294 23 Tashima, Y., Taguchi, R., Murata, C., Ashida, H., Kinoshita, T. and Maeda, Y. (2006) PGAP2 is essential for correct processing and stable expression of GPI-anchored proteins. Mol. Biol. Cell. 17, 1410-1420

24 Bosson, R., Jaquenoud, M. and Conzelmann, A. (2006) GUP1 of Saccharomyces cerevisiae encodes an O-acyltransferase involved in remodeling of the GPI anchor. Mol. Biol. Cell. 17, 2636-2645

25 Maeda, Y., Tashima, Y., Houjou, T., Fujita, M., Yoko-o, T., Jigami, Y, Taguchi, R. and Kinoshita, T. (2007) Fatty acid remodeling of GPI-anchored proteins is required for their raft association. Mol. Biol. Cell. 18, 1497-1506

26 Jaquenoud, M., Pagac, M., Signorell, A., Benghezal, M., Jelk, J., Bütikofer, P. and Conzelmann, A. (2008) The Gup1 homologue of Trypanosoma brucei is a GPI glycosylphosphatidylinositol remodelase. Mol. Microbiol. 67, 202-212

27 Roberts, W. L., Myher, J. J., Kuksis, A., Low, M. G. and Rosenberry, T. L. (1988) Lipid analysis of the glycoinositol phospholipid membrane anchor of human erythrocyte acetylcholinesterase. J. Biol. Chem. 263, 18766-18775

28 Bütikofer, P., Kuypers, F. A., Shackleton, C., Brodbeck, U. and Stieger, S. (1990)

Molecular species analysis of the glycosylphosphatidylinositol anchor of Torpedo marmorata acetylcholinesterase. J. Biol. Chem. 265, 18983-18987

29 Bütikofer, P., Zollinger, M. and Brodbeck, U. (1992) Alkylacyl

glycerophosphoinositol in human and bovine erythrocytes. Molecular species composition and comparison with glycosyl-inositolphospholipid anchors of erythrocyte acetylcholinesterases. Eur. J. Biochem. 208, 677-683

30 Treumann, A., Lifely, M. R., Schneider, P. and Ferguson, M. A. J. (1995) Primary structure of CD52. J. Biol. Chem. 270, 6088-6099

31 Brewis, I. A., Ferguson, M. A., Mehlert, A., Turner, A. J. and Hooper, N. M. (1995) Structures of the glycosyl-phosphatidylinositol anchors of porcine and human renal membrane dipeptidase. Comprehensive structural studies on the porcine anchor and interspecies comparison of the glycan core structures. J. Biol. Chem. 270, 22946-22956

32 Bayne, R. A. L., Kilbride, E. A., Lainson, F. A., Tetley, L. and Barry, J. D. (1993) A major surface antigen of procyclic stage Trypanosoma congolense. Molecular and biochemical parasitology. 61, 295-310 
33 Beecroft, R. P., Roditi, I. and Pearson, T. W. (1993) Identification and characterization of a major surface glycoprotein from procyclic stage Trypanosoma congolense. Molecular and biochemical parasitology. 61, 285-294

34 Utz, S., Roditi, I., Kunz Renggli, C., Almeida, I. C., Acosta-Serrano, A. and Bütikofer, P. (2006) Trypanosoma congolense procyclins: unmasking cryptic major surface glycoproteins in procyclic forms. Eukaryot. Cell. 5, 1430-1440

35 Bütikofer, P., Vassella, E., Boschung, M., Kunz Renggli, C., Brun, R., Pearson, T. W. and Roditi, I. (2002) Glycosylphosphatidylinositol-anchored surface molecules of Trypanosoma congolense insect forms are developmentally regulated in the tsetse fly. Molecular and biochemical parasitology. 119, 7-16

36 Sakurai, T., Sugimoto, C. and Inoue, N. (2008) Identification and molecular characterization of a novel stage-specific surface protein of Trypanosoma congolense epimastigotes. Molecular and biochemical parasitology. 161, 1-11

37 Thomson, L. M., Lamont, D. J., Mehlert, A., Barry, J. D. and Ferguson, M. A. J. (2002) Partial structure of glutamic acid and alanine-rich protein, a major surface glycoprotein of the insect stages of Trypanosoma congolense. J. Biol. Chem. 277, 4889948904

38 Greganova, E., Bütikofer, P. and Acosta-Serrano, A. (2010) The protease resistant surface (PRS) glycoconjugate from Trypanosoma congolense has an inositol-acylated glycosylphosphatidylinositol anchor, containing a significant proportion of myristate at the sn-2 position. Molecular and biochemical parasitology. 171, 50-54

39 Vassella, E., Bütikofer, P., Engstler, M., Jelk, J. and Roditi, I. (2003) Procyclin null mutants of Trypanosoma brucei express free glycosylphosphatidylinositols on their surface. Mol. Biol. Cell. 14, 1308-1318

40 Brun, R. and Schonenberger. (1979) Cultivation and in vitro cloning or procyclic culture forms of Trypanosoma brucei in a semi-defined medium. Short communication. Acta Trop. 36, 289-292

41 Bütikofer, P., Ruepp, S., Boschung, M. and Roditi, I. (1997) 'GPEET' procyclin is the major surface protein of procyclic culture forms of Trypanosoma brucei brucei strain 427. Biochem. J. 326, 415-423

42 Bütikofer, P., Boschung, M., Brodbeck, U. and Menon, A. K. (1996)

Phosphatidylinositol hydrolysis by Trypanosoma brucei glycosylphosphatidylinositol phospholipase C. J. Biol. Chem. 271, 15533-15541 
43 Field, M. C. and Menon, A. K. (1992) Biosynthesis of glycosylphosphatidylinositol membrane anchors. In Lipid Modification of Proteins: A Practical Approach (Hooper, N. M., Turner, A. J. and editors, eds.). pp. 155-190, IRL Press, Oxford

44 Priest, J. W., Mehlert, A., Arrowood, M. J., Riggs, M. W. and Ferguson, M. A. (2003) Characterization of a low molecular weight glycolipid antigen from Cryptosporidium parvum. J. Biol. Chem. 278, 52212-52222

45 Hsu, F. F. and Turk, J. (2000) Characterization of phosphatidylinositol,

phosphatidylinositol-4-phosphate, and phosphatidylinositol-4,5-bisphosphate by electrospray ionization tandem mass spectrometry: a mechanistic study. J. Am. Soc. Mass. Spectrom. 11, 986-999

46 Mayor, S., Menon, A. K. and Cross, G. A. M. (1991) Transfer of glycosylphosphatidylinositol membrane anchors to polypeptide acceptors in a cell-free system. J. Cell Biol. 114, 61-71

47 Fujita, M. and Kinoshita, T. (2009) Structural remodeling of GPI anchors during biosynthesis and after attachment to proteins. FEBS Lett

48 Sipos, G., Reggiori, F., Vionnet, C. and Conzelmann, A. (1997) Alternative lipid remodelling pathways for glycosylphosphatidylinositol membrane anchors in Saccharomyces cerevisiae. EMBO J. 16, 3494-3505

49 Tanaka, S., Maeda, Y., Tashima, Y. and Kinoshita, T. (2004) Inositol deacylation of glycosylphosphatidylinositol-anchored proteins is mediated by mammalian PGAP1 and yeast Bst1p. J. Biol. Chem. 279, 14256-14263

50 Güther, M. L., Leal, S., Morrice, N. A., Cross, G. A. and Ferguson, M. A. (2001) Purification, cloning and characterization of a GPI inositol deacylase from Trypanosoma brucei. EMBO J. 20, 4923-4934

51 Hong, Y., Nagamune, K., Morita, Y. S., Nakatani, F., Ashida, H., Maeda, Y. and Kinoshita, T. (2006) Remoyal or maintenance of inositol-linked acyl chain in glycosylphosphatidylinositol is critical in trypanosome life cycle. J. Biol. Chem. 281, 1159511602 


\section{Figure legends}

\section{Figure 1: Schematic representation of the major GPI-anchored glycoconjugates on the} surface of T. congolense procyclic and epimastigote forms. The lipid moieties of GARP [37], EPGENGT procyclin [34] and PRS [38] are shown in detail; the structure of the CESP lipid anchor is unknown. While GARP is heavily modified with phosphoglycans, the presence of this modification in EPGENGT procyclin is uncertain. Except for GARP, nothing is known about the structures of the GPI glycan cores of these molecules and, thus, the sequences shown are hypothetical. PRS is likely to be a GPI-anchored polysaccharide [35].

Figure 2: Identification of $\left[{ }^{3} \mathbf{H}\right]$-labelled GPI lipids. A, T. brucei and T. congolense Kilifi and Savannah procyclic forms $\left(0.5-1.0 \times 10^{9}\right.$ cells) were incubated in the presence of $\left[{ }^{3} \mathrm{H}\right]$ myristate for $40 \mathrm{~h}$ and GPI-anchored molecules were extracted into $9 \%$ butan-1-ol. $\left[{ }^{3} \mathrm{H}\right]-$ labelled lipids were released from labelled butan-1-ol extracts by treatment with GPI-PLD (left panel) or PI-PLC (right panel), partitioned between butan-1-ol and water, and analyzed by TLC using solvent system 1. Radiolabelled products (20-50’000 cpm) were visualized by fluorography. The migration of PA and lyso-PA standards run on the same plate is indicated. $B$, Time course of $\left[{ }^{3} \mathrm{H}\right]$-incorporation into GPIs. GPI-anchored molecules from $T$. congolense Savannah and Kilifi procyclic forms were labelled with $\left[{ }^{3} \mathrm{H}\right]$ myristate for $6-23 \mathrm{~h}$ and extracted and treated with GPI-PLD as described in A. Radiolabelled products (40-60'000 cpm) were analyzed by TLC in solvent system 1, visualized by fluorography, and quantified by scanning the lanes with a radioisotope detector. The numbers represent the relative amounts of radioactivity in the PA and lyso-PA spots of each lane; no other labelled products were detected (see also $A$ ).

Figure 3: Characterization of $\left[{ }^{3} \mathbf{H}\right]$-labelled GPI lipids. T. congolense Savannah procyclic forms were labelled with $\left[{ }^{3} \mathrm{H}\right]$ myristate for 6 or $48 \mathrm{~h}$ and extracted as described in Fig. 2. A, GPI-anchored molecules in the butan-1-ol extracts were treated with GPI-PLD (PLD), PIPLC (PLC) or nitrous acid (NA) and the released products analyzed by TLC using solvent system 1. The migration of PA, lyso-PA and PI standards run on the same plate is indicated. $B$, The products after PI-PLC treatment, migrating with the solvent front in $A$, were scraped and re-extracted from the plate and separated by TLC in solvent system 2 . The migration of diacylglycerol (DAG) and monoacylglycerol (MAG) standards run on the same plate is indicated. 
Figure 4: Analysis of protein-bound GPIs after pulse-chase labelling with $\left[{ }^{3} \mathbf{H}\right]$ myristate. T. congolense Savannah (left panel) and T. brucei (right panel) procyclic forms were labelled with $\left[{ }^{3} \mathrm{H}\right]$ myristate for $2 \mathrm{~h}$ (pulse). Subsequently, cells were washed to remove unincorporated label and the incubation was continued in the absence of label (chase). After 0, 4 and 22 h, GPI-anchored molecules were extracted as described in Fig. 2 and [ $\left.{ }^{3} \mathrm{H}\right]-$ labelled products released by GPI-PLD were analyzed by TLC using solvent system 1 . The migration of PA and lyso-PA standards run on the same plate is indicated.

Figure 5: Negative-ion ESI-MS-CID-MS analyses of PI moieties of whole butan-1-ol extracts. $A$, negative-ion ESI-mass spectra of PI species obtained after nitrous acid deamination of GPI molecules present in a butan-1-ol extract of parasites cultured for $6 \mathrm{~h}$. A similar spectrum was obtained from a butan-1-ol extract from parasites cultured for $48 \mathrm{~h}$ (see Figs. S1-S3). The ions at $\mathrm{m} / \mathrm{z}, 956$ and 1048 are phospholipid contaminants also present in the last butanol wash before nitrous acid deamination (not shown). $B$, proposed structures of the most abundant PI moieties.

\section{Figure 6: Incorporation of $\left[{ }^{3} \mathrm{H}\right]$ myristate into free GPIs of $T$. brucei EP/GPEET KO} cells. EP/GPEET KO parasites $\left(8-0.2 \times 10^{8}\right.$ cells) were incubated in the presence of $\left[{ }^{3} \mathrm{H}\right]$ myristate for 1-48 h, washed to remove unincorporated label, and delipidated with $\mathrm{CM}$ $(2: 1, v / v)$. Free GPIs were extracted with CMW (10:10:3, v/v/v), partitioned into the CMWaqu phase, and treated with GPI-PLD. Radiolabelled products were analyzed by TLC using solvent system 1 and visualized by fluorography. The lanes contain 4'200-8'200 cpm of labelled extracts.

Figure 7: Model of lipid remodelling reactions in protein- and polysaccharide-bound GPIs in T. congolense procyclic forms. See Discussion section for details. 
Table 1: Suggested identities of the released phosphatidylinositol species from GPIglycoconjugates present in the butanol extracts from $T$. congolense procyclic forms cultured for 6 and 48 hours.

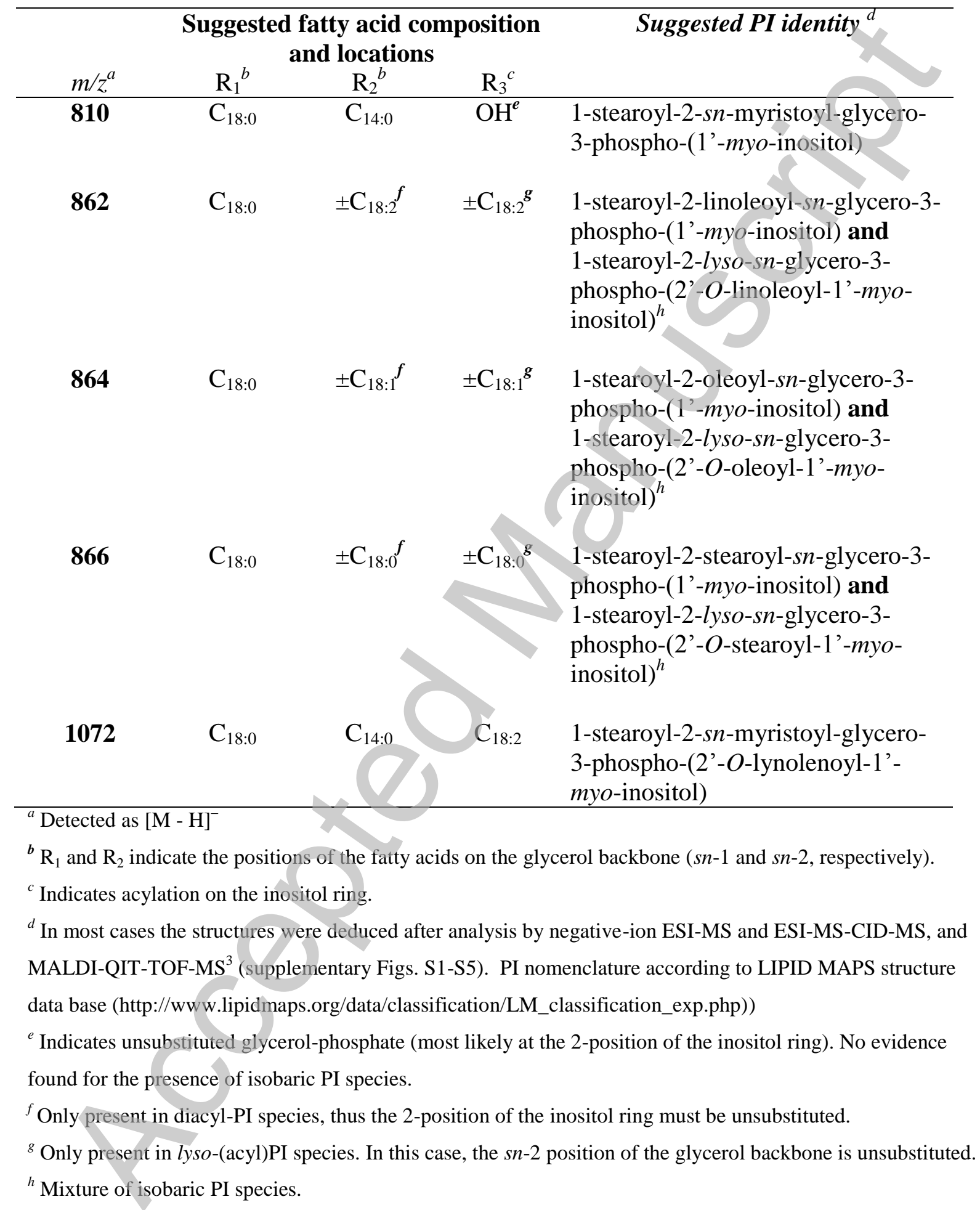


Figure 1

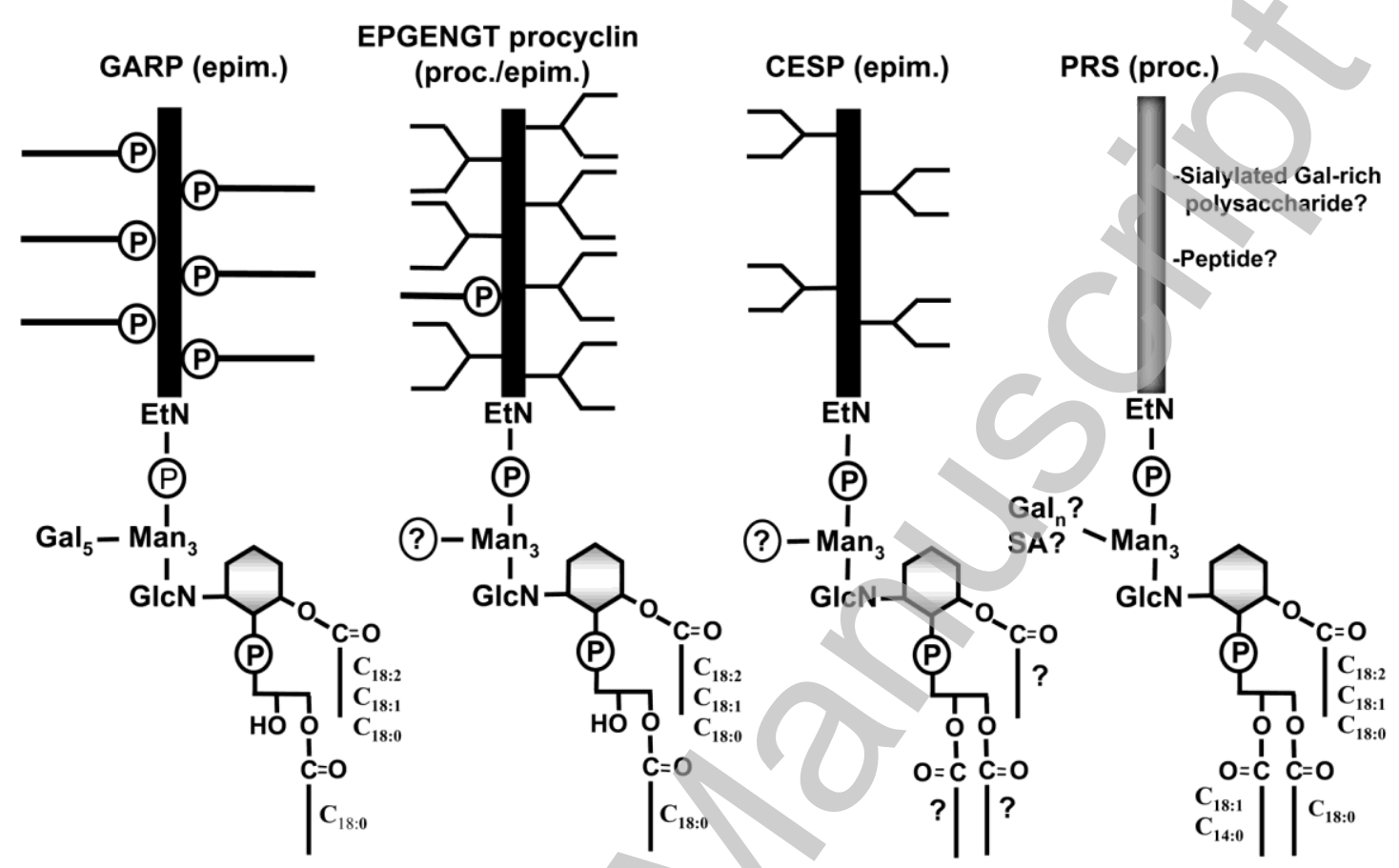

Licenced copy. Copying is not permitted, except with prior permission and as allowed by law.

(C) 2010 The Authors Journal compilation (c) 2010 Portland Press Limited 
Figure 2

A

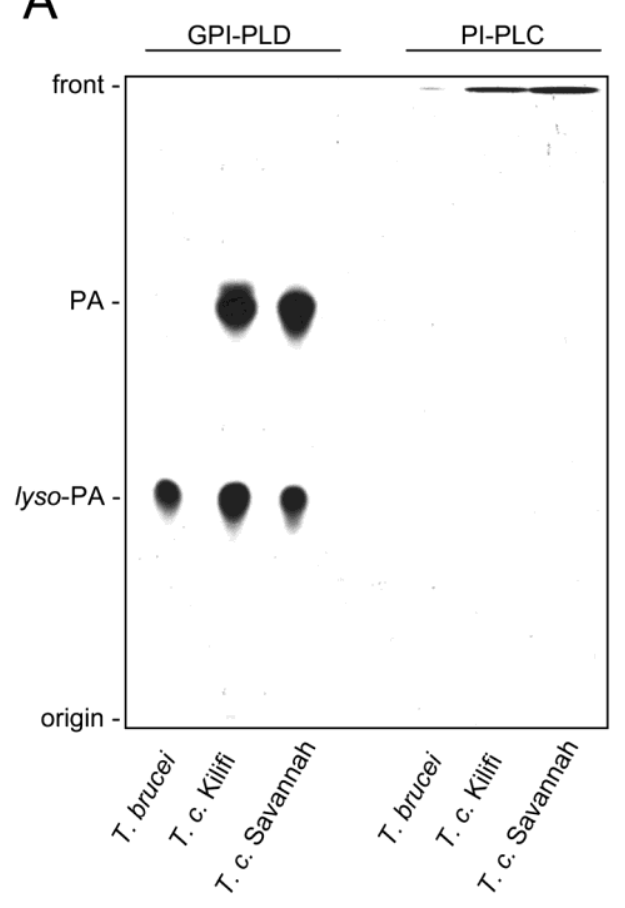

B

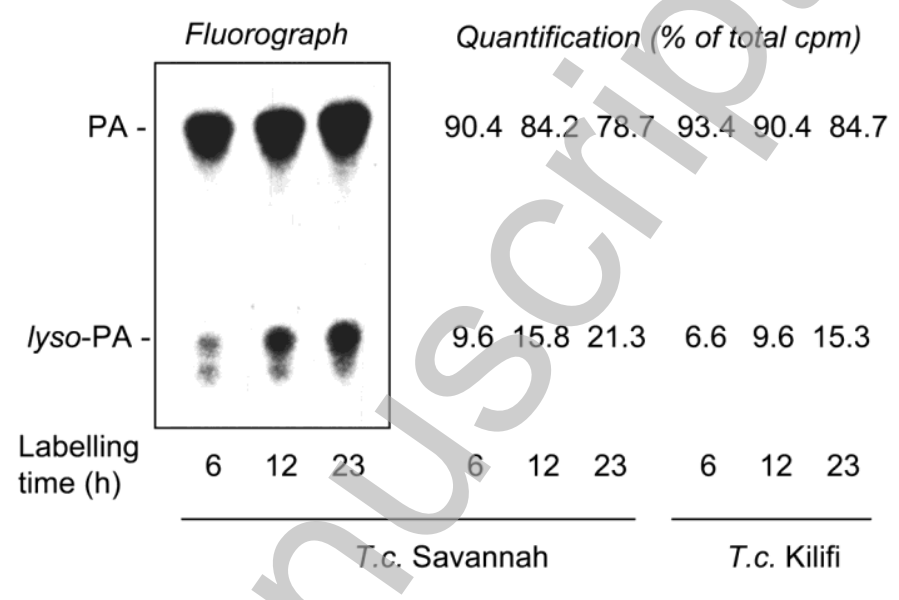


Figure 3

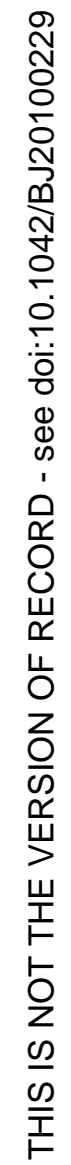

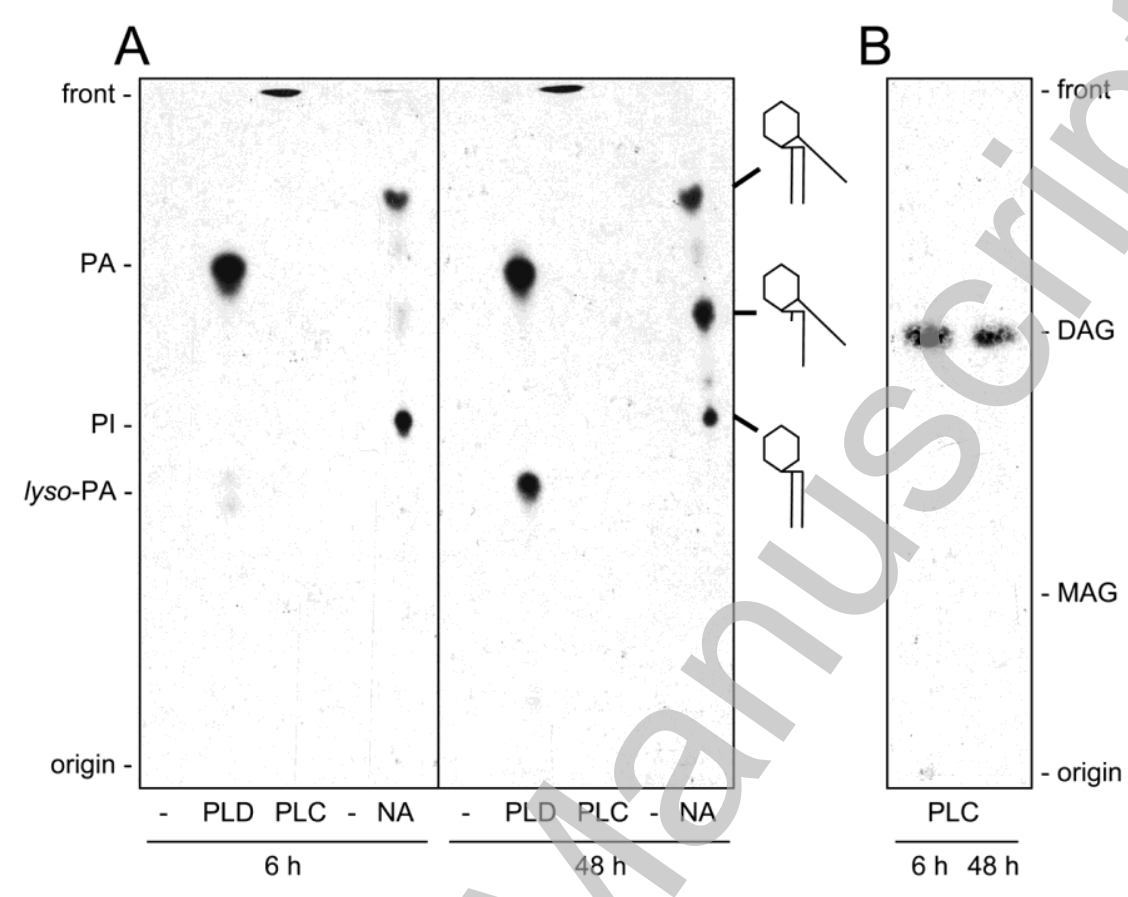

Licenced copy. Copying is not permitted, except with prior permission and as allowed by law. (C) 2010 The Authors Journal compilation (c) 2010 Portland Press Limited 
Figure 4

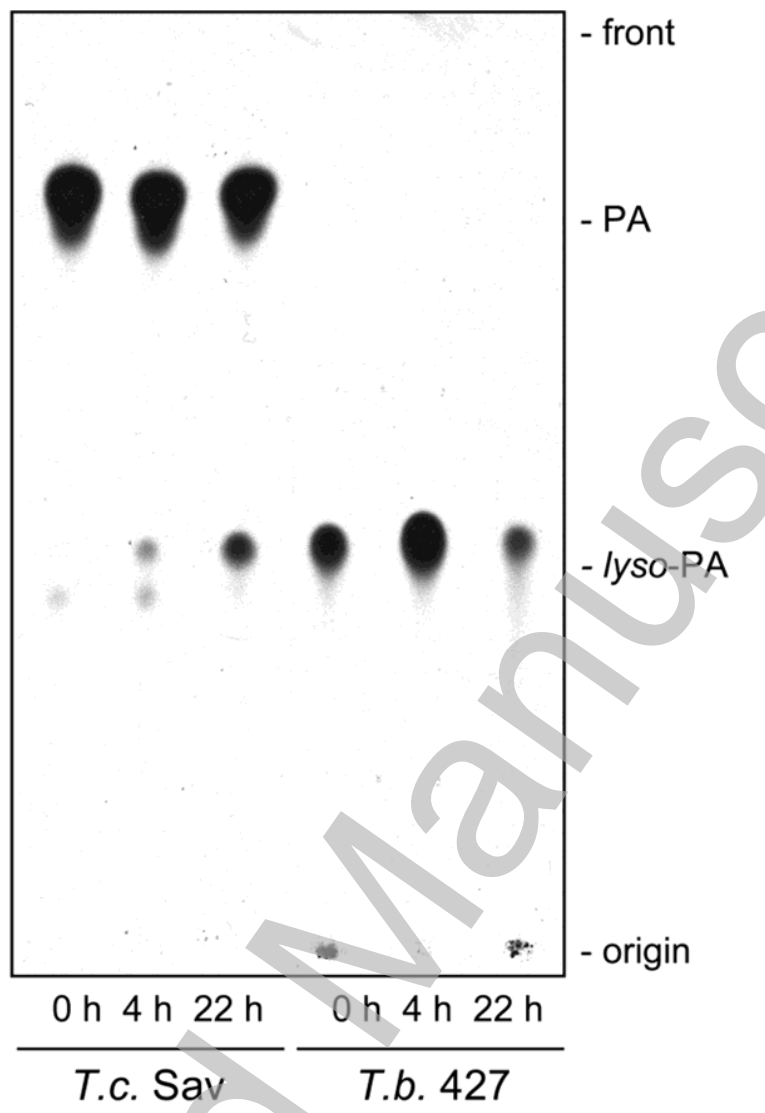


Figure 5

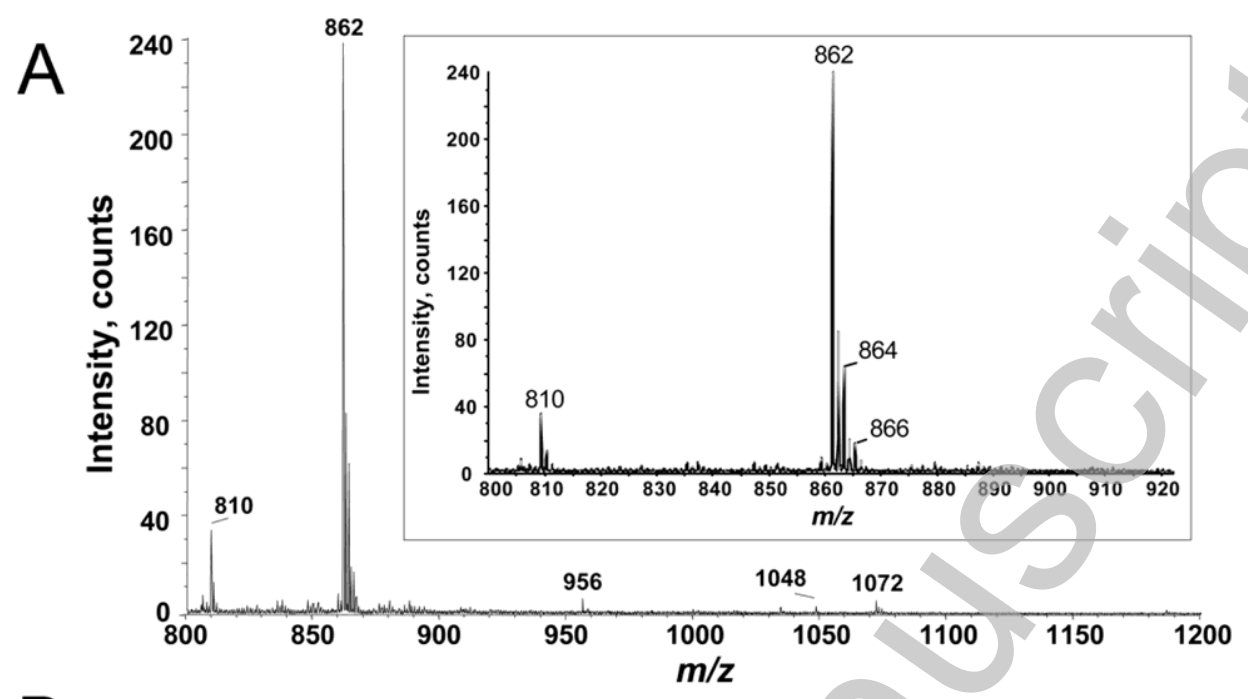

B
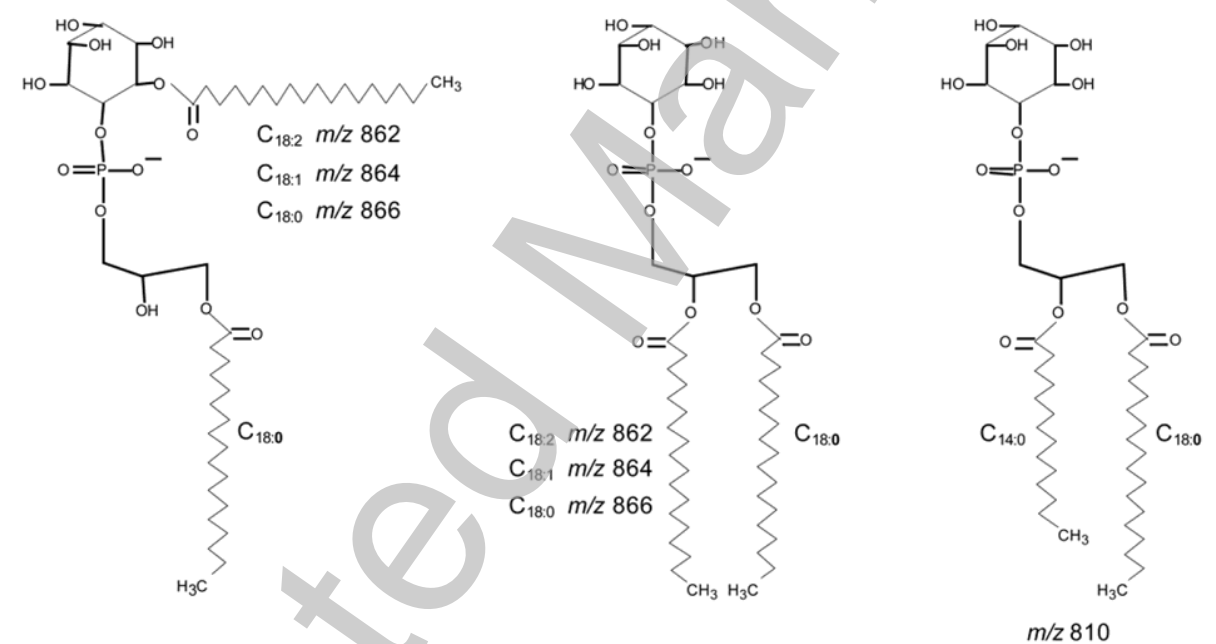

Licenced copy. Copying is not permitted, except with prior permission and as allowed by law. (C) 2010 The Authors Journal compilation (c) 2010 Portland Press Limited 
Figure 6

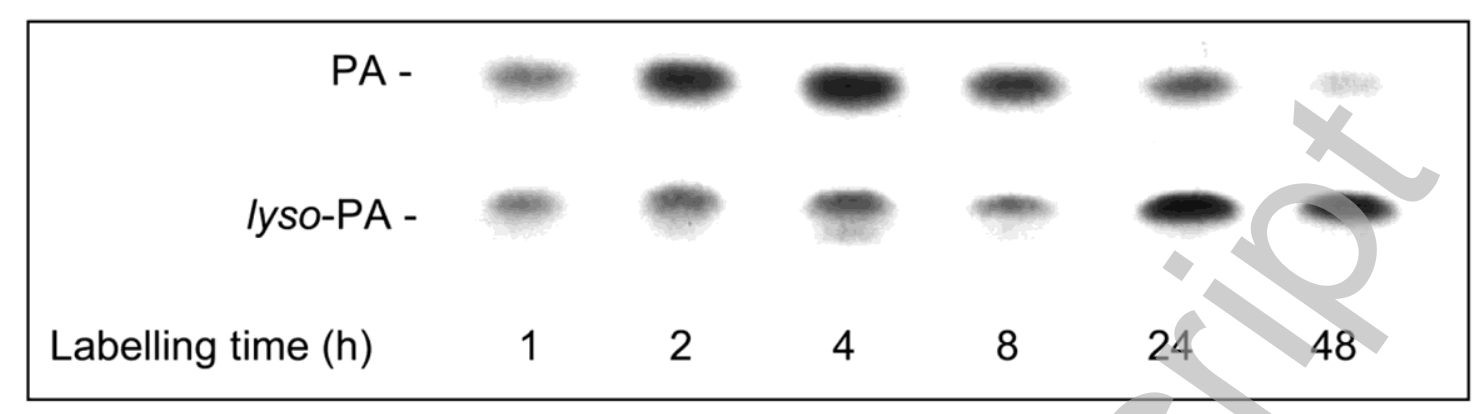


Figure 7

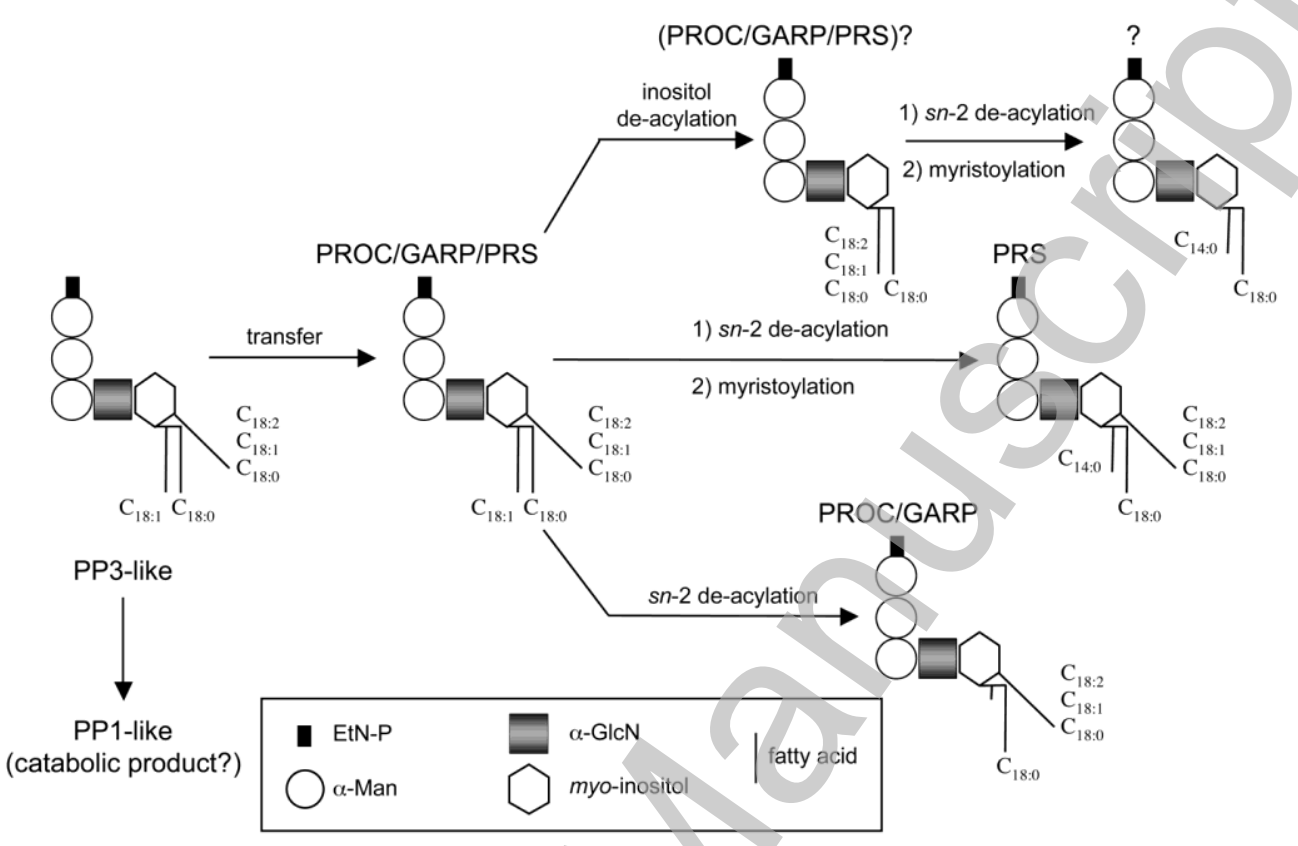

